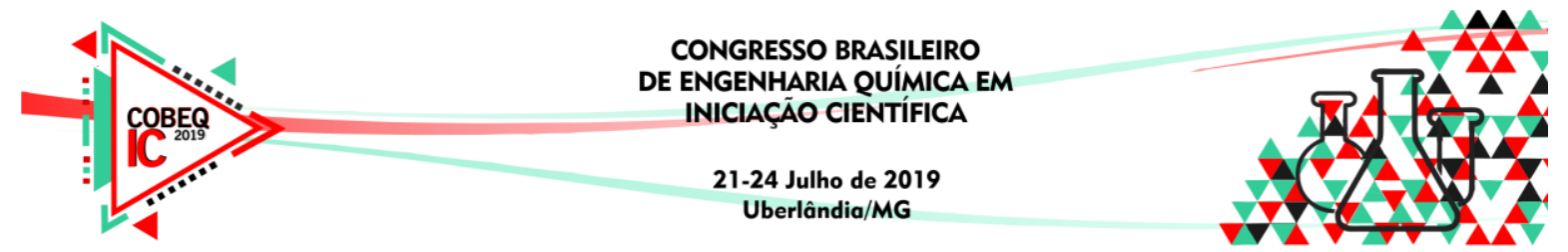

\title{
AVALIAÇÃO PRELIMINAR DA TROCA TÉRMICA EM UNIDADE PILOTO DE UM SISTEMA COILED TUBING
}

\author{
L. R. JUSTINO ${ }^{1}$, C. E. G. PEREIRA¹, B. R. OLIVEIRA ${ }^{1}$, E. C. H. PARAÍSO ${ }^{1}$, L. A. \\ CALÇADA $^{1}$ e C. M. SCHEID ${ }^{1}$ \\ ${ }^{1}$ Universidade Federal Rural do Rio de Janeiro \\ E-mail para contato: lorisjustino@gmail.com
}

\begin{abstract}
RESUMO - O abandono de poço é realizado quando não há mais exploração de petróleo naquele local, ou quando a área apresenta riscos ao meio ambiente. Para isto, diferentes tipos de fluidos são bombeados no poço, dentre eles a pasta de cimento. Este bombeamento pode ser feito por um sistema coiled tubing, que é um tubo de aço longo e flexível enrolado em um carretel. Sabe-se que durante o escoamento, diferentes trocas de calor afetam a temperatura do fluido, sendo importante prever e controlar esta variação a fim de que o cimento não endureça antes do tempo previsto. Este trabalho tem como objetivo avaliar a troca térmica no escoamento em coiled tubing através de experimentos em uma unidade piloto e a partir dos resultados propor e validar um modelo que possa prever a variação de temperatura. Uma unidade experimental foi construída de forma semelhante ao caso real. Experimentos foram realizados utilizando a água, a uma temperatura de $45^{\circ} \mathrm{C}$ e vazões de 0,20 e $0,65 \mathrm{~m}^{3} / \mathrm{h}$, bombeados em coiled tubing de $380 \mathrm{~m}$ durante aproximadamente 3 horas. As temperaturas foram aquisitadas, e a partir delas um modelo foi proposto considerando a troca de calor por atrito, por convecção natural e por radiação.
\end{abstract}

\section{INTRODUÇÃO}

Sabe-se que na indústria de petróleo, todo poço construído um dia será abandonado seja para evitar um dano ambiental ou quando sua exploração não é mais economicamente viável. Utilizado em diversas aplicações na área do petróleo, o sistema coiled tubing é composto por uma bobina e um tubo de aço enrolado longo e flexível, onde parte do tubo fica enrolado em um carretel, enquanto a outra parte é direcionada ao poço. Neste tubo, bombeia-se água, pasta de cimento e fluido de deslocamento, que são fluidos fundamentais para o abandono do poço. Este é feito por meio de tampões de cimento em diversas profundidades, onde pasta de cimento é bombeada através da parte enrolada do sistema, em que há troca de calor entre o fluido e o ambiente. Torna-se de suma importância o estudo da troca térmica de fluidos em um sistema coiled tubing, principalmente no escoamento de pastas de cimento. Dessa forma será possível evitar que a mesma cure antes do tempo previsto (Pereira, 2018). A Figura 1, apresenta um sistema coiled tubing.

Neste trabalho, pretende-se prever a troca térmica entre o fluido e o ambiente assim como a troca gerada pelo atrito do fluido ao escoar. Para isso experimentos foram conduzidos em uma 


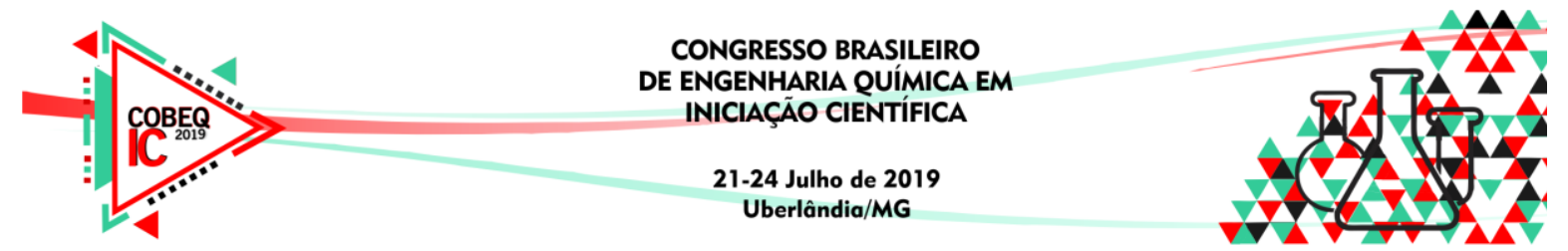

unidade piloto e um balanço de energia foi proposto com o intuito de avaliar a variação de temperatura na entrada e na saída do sistema.

Figura 1 - Sistema coiled tubing.

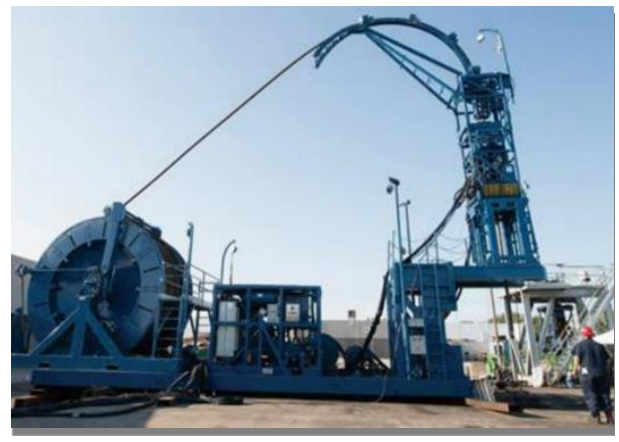

\section{MATERIAIS E MÉTODOS}

Neste trabalho, buscou-se construir uma unidade piloto que fosse correspondente ao caso real. Utilizou-se uma bobina com tubo de cobre enrolado, que por sua vez possui uma similaridade com o caso real. Essa bobina possui $375,8 \mathrm{~m}$ de comprimento divididos em 8 camadas onde em cada camada há medições de temperatura e pressão. Para determinar as pressões e temperaturas utiliza-se, respectivamente, transmissores de pressão e termopares, que medem a temperatura do fluido, do tubo e do ambiente. A Figura 2 mostra o sistema coiled tubing e um esquema da unidade experimental completa.

Figura 2 - a) Sistema coiled tubing da unidade piloto e b) unidade experimental.
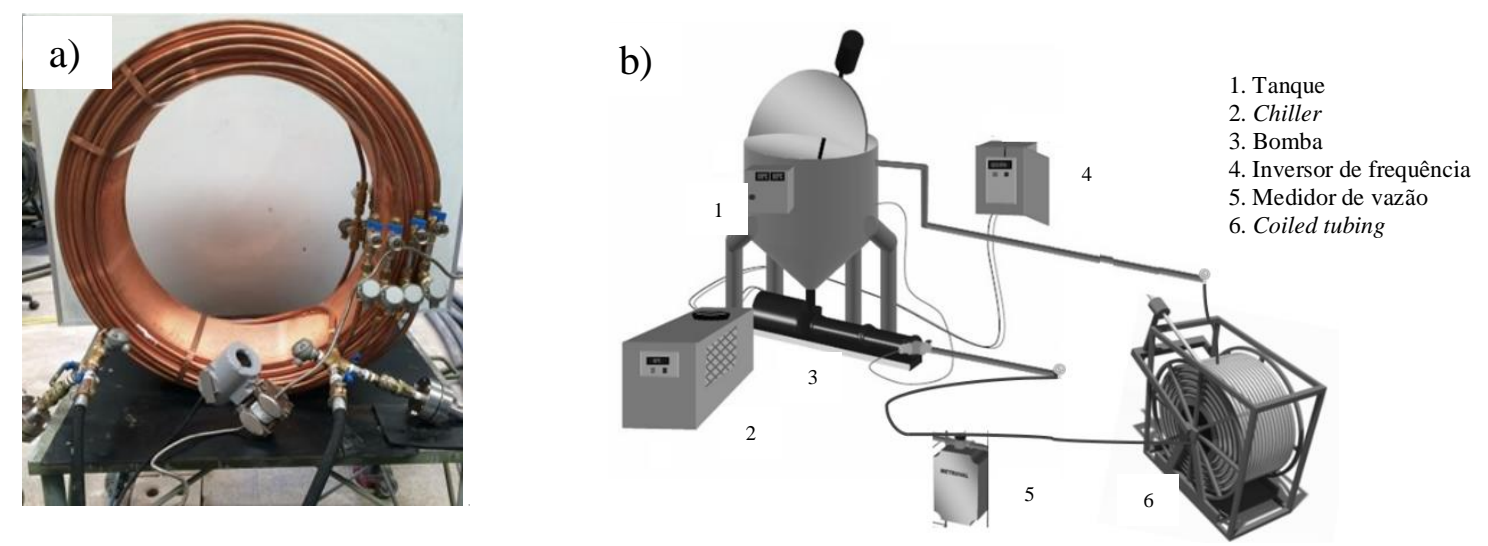

O fluido é preparado em um tanque que possui uma camisa e um chiller para controle da temperatura inicial. O coiled tubing é acoplado a um sistema de bombeio composto por uma bomba de deslocamento positivo. Sendo assim, o fluido é bombeado em um sistema fechado retornando para o tanque. Os dados de pressão, temperatura e vazão são aquisitados por um software sendo possível acompanhar os resultados em tempo real.

Os experimentos foram realizados utilizando como fluido a água a uma temperatura inicial constante de $45^{\circ} \mathrm{C}$ e temperatura ambiente de aproximadamente $25^{\circ} \mathrm{C}$. A água foi 


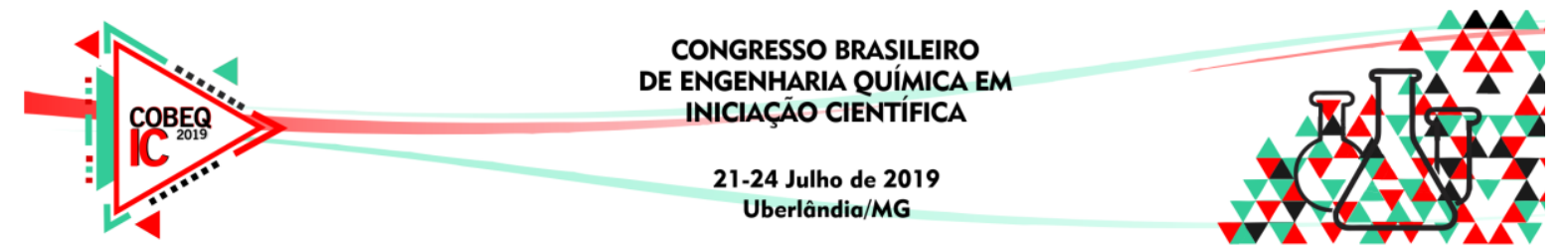

escolhida por ser um fluido Newtoniano, com propriedades físico-químicas familiares e por ter viscosidade modificada somente pela temperatura. $\mathrm{O}$ fluido foi bombeado em duas diferentes vazões $\left(0,2\right.$ e $\left.0,65 \mathrm{~m}^{3} / \mathrm{h}\right)$ a fim de se avaliar a influência da vazão na troca térmica, assim como o atrito gerado. Os dados foram aquisitados a cada minuto durante 3 horas.

A fim de se estudar a troca térmica ao longo do coiled tubing, um balanço de energia foi proposto. O calor sensível para alterar a temperatura na entrada e saída do sistema é afetado por duas formas de troca de calor: o calor recebido por atrito e o que é perdido por convecção natural e por radiação. A radiação térmica foi considerada visto que todos os corpos emitem radiações térmicas proporcionais às suas temperaturas. As Equações 1 e 2 representam o balanço de energia proposto.

$$
\begin{gathered}
q_{\text {sensível }}=q_{\text {atrito }}-\left(q_{\text {convecção natural }}+q_{\text {radiação }}\right) \\
\rho Q C p\left(T_{f_{\text {sai }}}-T_{f_{\text {entra }}}\right)=\Delta P \cdot Q-\left[\sum \overline{h_{e}} A\left(T_{t_{\text {média }}}-T_{a m b}\right)+\sigma \varepsilon A\left(T_{t_{\text {média }}}^{4}-T_{a m b}^{4}\right)\right]
\end{gathered}
$$

sendo $q_{\text {sensível }}$ a taxa de calor sensível (W), qatrito a taxa de calor por atrito (W),

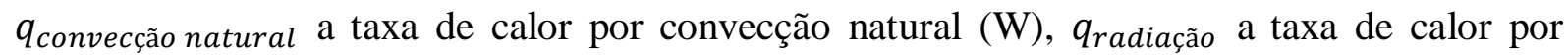
radiação (W). Na Equação 2, tem-se $\rho$ a densidade do fluido $\left(\mathrm{kg} / \mathrm{m}^{3}\right), Q$ a vazão volumétrica $\left(\mathrm{m}^{3} / \mathrm{s}\right), c p$ calor específico do fluido (J/kg.K), $T f_{\text {sai }}$ temperatura de saída do fluido (K), $T$ fentra temperatura de entrada do fluido $(\mathrm{K}), \Delta P$ queda de pressão total $(\mathrm{Pa}), \overline{h_{e}}$ coeficiente convectivo de troca térmica médio externo $\left(\mathrm{W} / \mathrm{m}^{2} \mathrm{~K}\right), A$ área de troca térmica $\left(\mathrm{m}^{2}\right), T$ tmédia temperatura média do tubo de cobre (K), Tamb temperatura ambiente (K), $\sigma$ constante de Stefan Boltzmann (5,67x10$\left.{ }^{8} \mathrm{~W} / \mathrm{m}^{2} \mathrm{~K}^{4}\right), \varepsilon$ emissividade do cobre $(0,6$ adimensional $)$.

Como o sistema possui diversas faces, foi necessário avaliar a troca térmica separadamente em cada uma delas. Para isso, fez-se uma consideração de que o coiled tubing é um cilindro maciço com uma região anular onde a área externa e interna do cilindro foi estendida. Correlações de Nusselt $(\mathrm{Nu})$ foram utilizadas para o cálculo do coeficiente convectivo de calor (h) segundo a Equação 3. As direções de troca térmica consideradas, assim como as correlações de Nusselt, faixa de aplicação, comprimento característico e área de troca térmica correspondentes às faces estão representadas abaixo. As correlações foram propostas Churchill e Chu (1975) apud Bergman et al. (2014).

$\overline{N u}=\frac{\bar{h} L_{c}}{k_{f}}$

Escoamento externo em cilindro horizontal (Figura 3 e Equações de 4 a 6):

Figura 3 - Esquema do escoamento externo em cilindro horizontal.

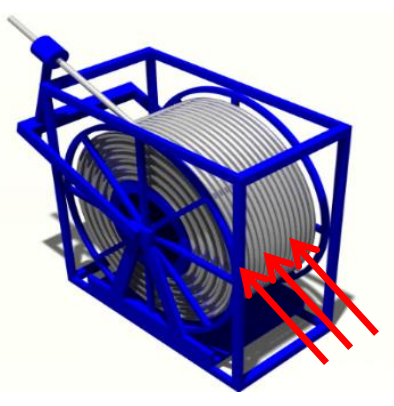

$$
\begin{aligned}
& \overline{N u_{D}}=\left\{0,60+\frac{0,387 R a_{D}^{1 / 6}}{\left[1+\left(0,559 / P_{r}\right) 9 / 16\right] 8 / 27}\right\} 2 \\
& L_{c}=D_{e e} \\
& A_{c}=2 \pi R_{e e} L_{e}
\end{aligned}
$$




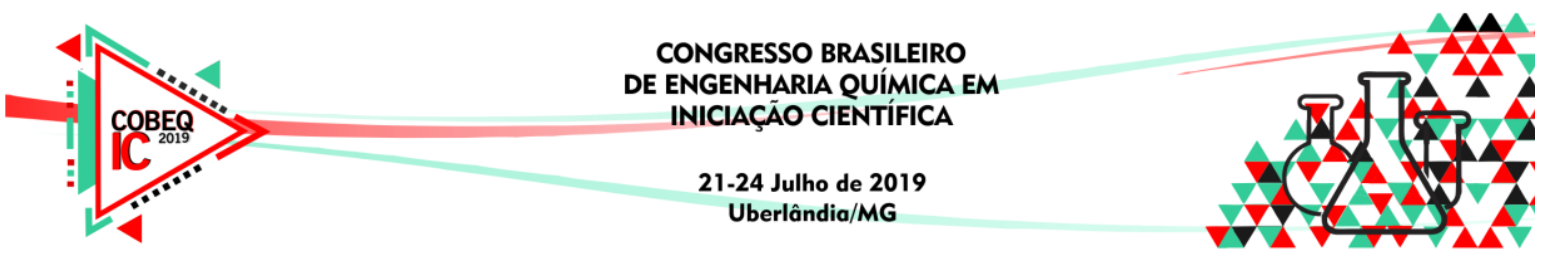

Escoamento em placa vertical nas duas faces (Figura 4 e Equações de 7 a 9):

Figura 4 - Esquema do escoamento em placa vertical.

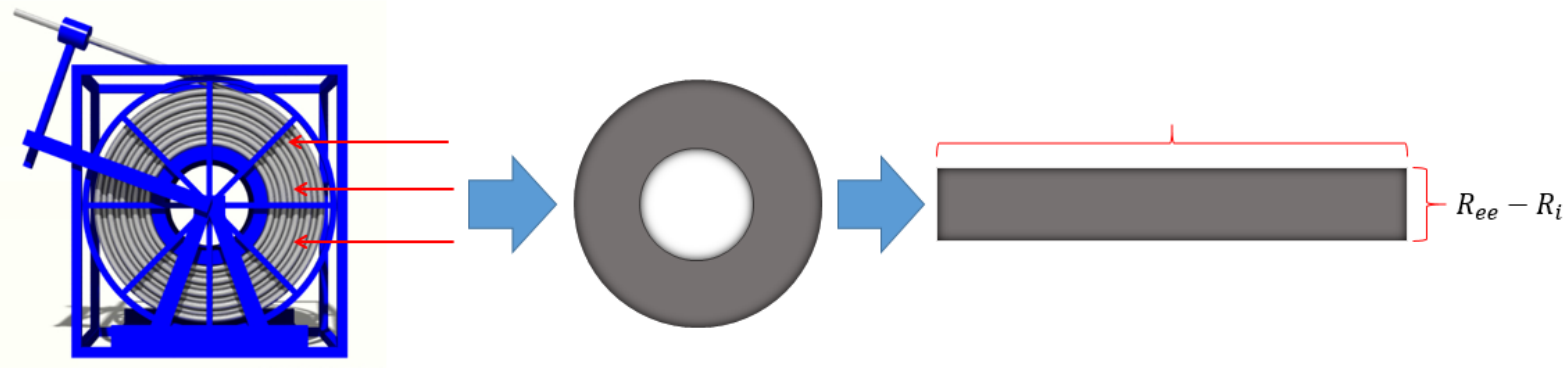

$\overline{N u}_{L}=\left\{0,825+\frac{0,387 R a_{L}^{1 / 6}}{\left[1+\left(0,492 / P_{r}\right) 9 / 16\right] 8 / 27}\right\} 2$

$L_{c}=R_{e e}-R_{i}$

$A_{L}=\pi\left(R_{e e}^{2}-R_{i}^{2}\right)$

Escoamento em placa plana horizontal (Figura 5 e Equações de 10 a 13):

Figura 5 - Esquema do escoamento em placa plana horizontal.

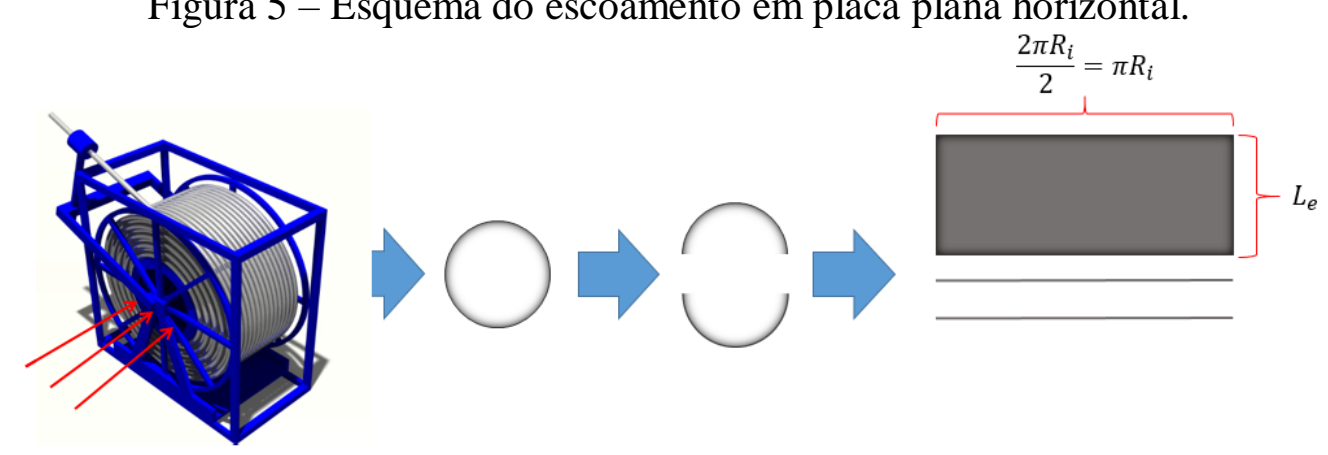

Superfície inferior: $\overline{N u}_{L}=0,15 R a_{L}^{1 / 3}$

Superfície superior: $\overline{N u}_{L}=0,52 R a_{L}^{1 / 5}$

$L_{c}=\frac{\text { área }}{\text { perímetro }}=\frac{\pi R_{i} L_{e}}{2\left(\pi R_{i}+L_{e}\right)}$

$A_{L}=\pi R_{i} L_{e}$

sendo $\bar{h}$ o coeficiente convectivo médio $\left(\mathrm{W} / \mathrm{m}^{2} \mathrm{~K}\right), \overline{N u_{L}}$ e $\overline{N u_{D}}$ o número de Nusselt médio, (adimensional), 1 o comprimento característico (m), $R a_{\mathrm{L}}$ e $R a_{\mathrm{D}}$ o número de Rayleigh 


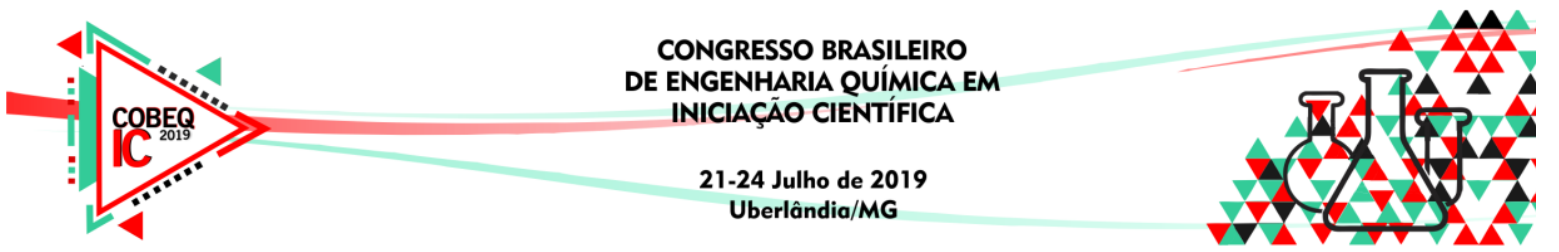

(adimensional), $\mathrm{P}_{\mathrm{r}}$ o número de Prandlt (adimensional), $\mathrm{L}_{\mathrm{c}}$ o comprimento característico $(\mathrm{m}), A_{C}$ e $A_{L}$ a área de troca térmica $\left(\mathrm{m}^{2}\right), R_{i}$ o raio interno do carretel $(\mathrm{m}), R e e$ o raio externo do carretel com comprimento estendido (m) e Le a largura do carretel com comprimento estendido (m).

A taxa de calor por convecção natural é apresentada na forma de somatório na Equação 2 devido às considerações feitas quanto à geometria da unidade. A Tabela 1 apresenta alguns dados utilizados nos cálculos deste trabalho.

Tabela 1 - Dados para cálculo da troca térmica

\begin{tabular}{|c|c|c|c|c|c|c|}
\hline $\begin{array}{c}\text { Densidade } \\
\text { da água } \\
\left(\mathrm{kg} / \mathrm{m}^{3}\right)\end{array}$ & $\begin{array}{c}\text { Calor } \\
\text { específico } \\
\text { da água } \\
(\mathrm{J} / \mathrm{kg} . \mathrm{K})\end{array}$ & $\begin{array}{c}\text { Viscosidade } \\
\text { da água a } \\
45^{\circ} \mathrm{C}(\mathrm{Pa} . \mathrm{s})\end{array}$ & $\begin{array}{c}\text { Condutividade } \\
\text { térmica da } \\
\text { água }(\mathrm{W} / \mathrm{m} . \mathrm{K})\end{array}$ & $\begin{array}{c}\text { Área } \\
\text { externa } \\
\left(\mathrm{m}^{2}\right)\end{array}$ & $\begin{array}{c}\text { Área } \\
\text { interna } \\
\left(\mathrm{m}^{2}\right)\end{array}$ & $\begin{array}{c}\text { Área } \\
\text { Lateral } \\
\left(\mathrm{m}^{2}\right)\end{array}$ \\
\hline 990 & 4180 & 0,0006 & 0,63 & 1,17 & 0,77 & 0,39 \\
\hline
\end{tabular}

\section{RESULTADOS E DISCUSSÕES}

Nos gráficos da Figuras 6 tem-se a variação da temperatura pelo tempo nas vazões de 0,2 e $0,65 \mathrm{~m}^{3} / \mathrm{h}$.

Figura 6 - Temperatura do fluido em todas as camadas com temperatura do fluido de entrada desejada de $45^{\circ} \mathrm{C}$ a) $0,20 \mathrm{~m}^{3} / \mathrm{h} \mathrm{e} \mathrm{b)} 0,65 \mathrm{~m}^{3} / \mathrm{h}$.
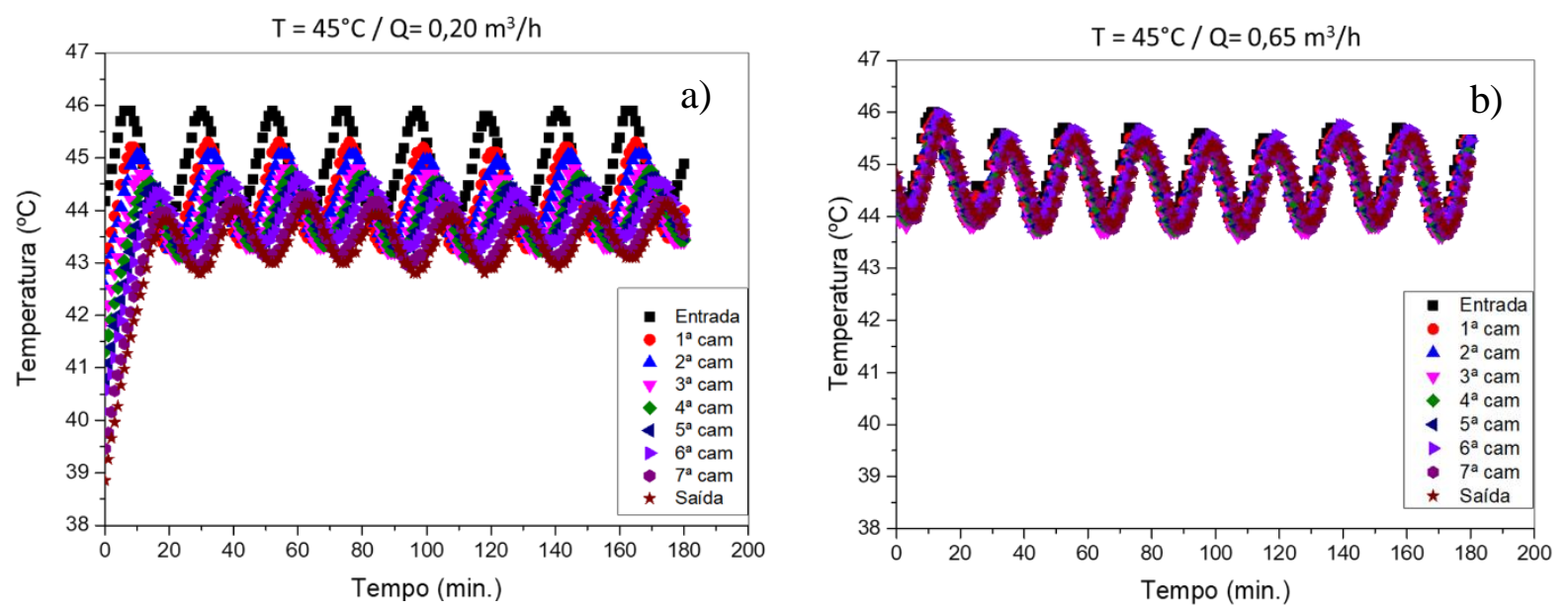

O gráfico tem um comportamento senoidal devido à dificuldade no controle de temperatura do fluido no tanque. Nota-se que quando a vazão é baixa a temperatura de saída é menor que a de entrada, enquanto que em vazão mais alta percebe-se que a temperatura na entrada e na saída são praticamente as mesmas. Isto revela que em uma alta vazão, a perda de calor é compensada pelo ganho de calor por atrito, o que não ocorre em uma baixa vazão.

A Tabela 2 apresenta os resultados obtidos pela modelagem proposta assim como o erro absoluto calculado pela diferença entre o calor sensível e a soma dos calores de atrito, 


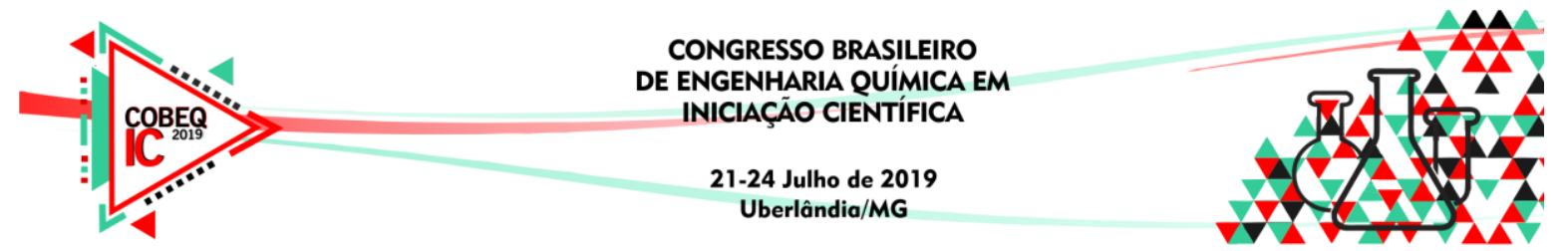

convecção e radiação calculados. O calor sensível foi calculado utilizando a diferença de temperatura experimental obtida. Vale ressaltar que devido às oscilações experimentais, foi feita uma média da temperatura em cada camada. Os resultados são: a taxa de calor sensível, atrito, convecção natural e radiação, e o erro absoluto para cada vazão.

Tabela 2 - Resultado do balanço de energia proposto para o experimento de $45^{\circ} \mathrm{C}$ nas duas vazões.

\begin{tabular}{|c|c|c|c|c|c|c|}
\hline $\begin{array}{c}Q \\
\left(\mathrm{~m}^{3} / \mathrm{h}\right)\end{array}$ & $\begin{array}{c}q_{\text {sensivel }} \\
(\mathrm{W})\end{array}$ & $\begin{array}{c}q_{\text {atrito }} \\
(\mathrm{W})\end{array}$ & $\begin{array}{c}q_{\text {conv ext }} \\
(\mathrm{W})\end{array}$ & $\begin{array}{c}q_{\text {rad }} \\
(\mathrm{W})\end{array}$ & $\begin{array}{c}q_{\text {atrito }}- \\
q_{\text {conv ext }}- \\
q_{\text {rad }}(\mathrm{W})\end{array}$ & $\begin{array}{c}\text { Erro } \\
\text { Absoluto } \\
(\%)\end{array}$ \\
\hline 0,20 & -292 & 10,74 & 109,63 & 124,44 & -225 & 23,1 \\
\hline 0,65 & -23 & 275,76 & 131,46 & 141,87 & -2 & 89,5 \\
\hline
\end{tabular}

Pode-se observar que o modelo não se ajusta aos dados experimentais, ainda que em uma menor vazão o erro obtido seja relativamente aceitável. Desta forma, deve-se reavaliar os termos considerados para o cálculo da variação da temperatura do fluido ao escoar.

\section{CONCLUSÃO}

A partir dos resultados, conclui-se a importância do estudo da troca térmica do fluido com o ambiente, bem como o ganho de calor por atrito e a perda de calor por convecção natural e radiação. O modelo proposto ainda não foi capaz de prever satisfatoriamente a variação de temperatura. Logo, pretende-se propor um novo balanço de energia reavaliando os termos considerados no cálculo da variação de temperatura do fluido ao escoar.

\section{REFERENCIAS}

BERGMAN, T. L.; LAVINE, A. S.; INCROPERA, F. P.; DEWITT, D. P. Fundamentos de Transferência de Calor e de Massa, 7 ed., Rio de Janeiro: LTC, Cap. 9, p. 386, 389, 390, 2014

CHURCHILL, S. W.; CHU, H. H. S. Int. J. Heat Mass Transfer, 18, p. 1049 e 1323, 1975

FERNANDES, F. A. N.; PIZZO, S. M.; MORAES Jr., D. Termodinâmica química, $1^{\text {a }}$ ed., 2006

PEREIRA, C. E. G. Estudo da Perda de Carga no Escoamento de Fluidos Newtonianos em Coiled Tubing. 2018. Dissertação (Mestrado em Engenharia Química) Instituto de Tecnologia, Universidade Federal Rural do Rio de Janeiro. 